\title{
Compression-dependency of soft tissue bioimpedance for in-vivo and in-vitro tissue testing
}

\author{
Sepideh M. Moqadam ${ }^{1}$, Parvind Grewal ${ }^{2}$, Majid Shokoufi ${ }^{1}$ and Farid Golnaraghi ${ }^{1,3}$ \\ 1. School of Mechatronic Systems Engineering, Simon Fraser University, Vancouver, Canada \\ 2. BC Cancer Agency, Abbotsford, Canada \\ 3. E-mail any correspondence to:mfgolnar@sfu.ca
}

\begin{abstract}
The present study determines the effect of compression over bioimpedance of healthy soft tissue (in-vitro and in-vivo). Electrical impedance spectroscopy (EIS) is a promising tissue characterization and tumor detection technique that uses tissue impedance or admittance to characterize tissue and identify tissue properties as well as cell structure. Variation in EIS measurements while applying pressure suggests that compression tends to affect soft tissue bioimpedance. Moreover, the displacements in tissue caused by applied compression may provide useful information about the structure and state of the tissue. Thus combining the changes to the electrical properties of tissue resulted by applied compression, with the changes in tissue displacements caused by applied compression, and consequently measuring the effect that electrical and mechanical properties have on each other, can be useful to identify tissue structure. In this study, multifrequency bioimpedance measurements were performed on in-vitro and invivo soft tissue at different pressure levels. Increasing compression on the in-vitro tissue results in an increase in both extracellular resistance and membrane capacitance while it causes a reduction in the intracellular resistance. However, as the compression over the in-vivo samples increases, the intracellular and extracellular resistance increase and the membrane capacitance decreases. The in-vivo measurements on human body are also tested on contralateral tissue sites and similar tissue impedance variation trends are observed in the contra-lateral sites of human body. The evidence from these tests suggests the possibility of using this EIS-Pressure combined measurement method to improve tumor detection in soft tissue. Based upon the observations, the authors envision developing an advanced model based upon the Cole model, which is dependent on tissue displacements.
\end{abstract}

Keywords: Bioimpedance, soft tissue, compression, in-vivo, invitro, electrical impedance spectroscopy

\section{Introduction}

Electrical and mechanical properties of tissue play a prominent role in tumor detection [1-4]. Much research has been performed independently on the mechanical and electrical properties of soft tissue and the effect that tissue pathology has on these properties. However, the link between mechanical and electrical properties of tissue and their correlation to pathological fluctuations remains unclear.

Pathology in soft tissue changes the stiffness of its local area [5]. Stiffness of soft tissue, which differs in tumor and its surrounding healthy tissue, can be estimated by measuring the applied external load to the body surface and its resultant displacement [6]. In the majority of breast examinations, palpation method is used to examine the breast [7], thus characterizing the compressive behavior of soft tissue provides insight into the mechanism of tissue pathology. Fischer introduced a device to quantify palpation of tissue in an objective manner [9]. He suggested the use of a tissue compliance meter in order to monitor the variation in the soft tissue modulus of elasticity when there is a malignancy in the tissue. Fung et al [8] identified the effect of pathological fluctuations on the elastic characteristics of the microscopic and macroscopic structural organization of tissue. Although these methods provide useful insight regarding the elastic features of tissue, some researchers looked into measuring the modulus elasticity of the tissue by means of imaging techniques. Many techniques have been proposed for examining abnormal breast tissue based on imaging the elastic contrast $[10,11]$. Most imaging techniques record the images of the tissue at two different applied forces and then the displacement of tissue is measured. Given the applied force and the displacement of soft tissue, its stiffness can be computed.

Soft tissue behavior is mostly close to the behavior of viscoelastic materials. However, in order to develop a mathematical model for behavior of soft tissue using a single elastic or shear modulus, most researchers have assumed that soft tissue is isotropic, elastic [12-15] and nearly incompressible [16]. Under these assumptions, Sarvazyan et al reported a study of 150 specimens of normal and cancerous tissues which showed that cancerous tissue can be as much as 7 times stiffer than normal tissue [14].

Besides, Fricke and Morse observed a higher capacitance in breast malignant tumors in comparison with the healthy breast [17]. Later in 1990, Morimoto et al studied the electrical impedance of breast tumors in-vivo and calculated the intracellular resistance, the extracellular resistance and cell membrane capacitance of normal and pathological tissue based on the impedance spectra at a frequency range of 0 to $300 \mathrm{kHz}$ and a circuit model [18]. They reported a statistically significant increase in the intraand extracellular resistances and a statistically significant decrease in the membrane capacitance in the pathological tissue. Jossinet et al performed an experiment on 64 
subjects with normal, benign and carcinoma breast tissues [19]. Jossinet compared the complex impedance loci of normal and carcinoma in a frequency range of $488 \mathrm{~Hz}$ to 1 $\mathrm{MHz}$ and an obvious difference was observed in the shape and location of the loci at frequencies above $125 \mathrm{kHz}$ for normal, benign and carcinoma tissue. Keshtkar et al performed electrical impedance measurements on 38 invivo benign and malignant subjects at a frequency range of 2- $348 \mathrm{kHz}$ [20]. The results revealed that the impeditivity of malignant subjects were significantly higher than the impeditivity of benign subjects which was in contrast to the results presented by Brown et al [21] and Gonzales-Correa [22].

Kim et al [23] developed a new analysis technique on the admittance of freshly-excised malignant breast tissue and its surrounding normal tissues which resulted in a clear distinction between the pathological and normal tissues. In a study conducted by Laufer et al [17], the Cole model, which is an electrical circuit equivalent model consisting of one resistor in parallel with a resistor and a constant phase element (CPE) which are in series [24], was fitted to the impedance data. The four parameters of the Cole model were compared in the cancerous and normal liver tissue. Obtaining the parameters of the Cole model gave a better understanding of the tissue in cellular level. The results illustrated that the conductivity of cancerous tissue was much higher than that of normal tissue.

Although many studies have focused on electrical and mechanical properties of healthy and cancerous tissue, the correlation of electrical and mechanical properties in healthy and cancerous tissue has not been established and compared yet.

Soft tissue electrical properties change due to tissue compression [25]. Different types of tissue have diverse compressive behaviors. Little within the literature has been published regarding the effect of compressive behavior of soft tissue on the changes of electrical properties. Gonzalez et al [26] measured the resistivity of the squamous and columnar tissues of three rats. The bioimpedance of these samples were measured under the applied pressure. They observed that by increasing the applied pressure not only the resistivity increases, but also the difference in the changes of resistivity of two types of tissue was sizeable. They suggest that the different resistivity of each type of tissue can be an indicator of its structure. Belmont's test [27] on a soft tissue-mimicking phantom showed a decrease in the admittance of the phantom under compression. They showed that tofu can be used as a suitable phantom for bioimpedance measurements under applied pressure. They established a correlation between the viscoelastic behavior of tofu and its electrical admittance. It was illustrated in this study that the impedance is influenced by the amount of conductive fluid within a porous material.

Jossinet et al examined the impedance changes in tissue exposed to high-energy ultrasound and observed a $20 \%$ increase in impedance magnitude at low frequency and a $30 \%$ decrease in impedance magnitude at high frequency
[28]. Dodde et al [25] studied the electrical properties of tissue under compression at the cellular level. A non-linear behavior was reported by Dodde at a compression level around $60 \%$. The findings of Dodde et al are discussed and compared to the results of this study in the discussion.

The purpose of the present study is to find the correlation between variations of tissue electrical properties with changes of displacements of soft tissue. We postulate that electrical properties of tissue are related to the mechanical properties and also any type of tissue has a specific electrical-mechanical properties based model. Since tumors in soft tissues change both the electrical and mechanical properties of tissue, it is postulated that healthy and tumorous tissues have different electrical-mechanical correlation. Thus, by finding this relationship and model, the structure, type and abnormality of the tissue can be estimated. Therefore, presence of inhomogeneity in the soft tissue electrical-mechanical behavior may help develop a tumor detection technique.

To test the validity of this claim, various types of invivo and in-vitro tissues are mechanically and electrically tested. As compared by [29], one model which fits to the measured data well is the Cole model [30, 31]. The modulus of elasticity of the tested tissues is calculated from the linear relationship of engineering stress-strain graphs. The effect that compression has on the electrical properties of tissue and also the relationship between electrical features and tissue displacement is elaborated in this paper.

\section{Materials and methods}

In this study, the bioimpedance and elastic modulus of twenty in-vitro chicken breast samples, two in-vitro rat breast samples and eleven in-vivo human subjects were measured. Ethics approval from the Office of Research Ethics, Simon Fraser University, was taken for collecting electrical and mechanical data of in-vivo human tissue. An advance approval of the animal care protocol was also obtained from the University Animal Care Committee of Simon Fraser University for in-vitro experiments. Details of the experimental setups, procedures and methods are given below.

\section{Experimental setup and procedure}

Bioimpedance and modulus of elasticity of in-vivo and invitro tissue were measured during electrical and mechanical experimental procedures by two different setups, which will be explained in the following sections.

Electrical impedance setup and procedure. Bioimpedance measurements of various types of tissue were conducted while incremental pressure levels were applied to the invivo and in-vitro tissue. For this experiment an impedance spectroscope HF2IS along with a transimpedance amplifier HF2TA (TA) by Zurich Instrument (Zurich Instrument Inc., Switzerland) were interfaced. The detailed electrical 
impedance system setup is explained by Grewal [32]. The bioimpedance measurements were conducted using a two point measurement technique $[33,34]$ at 50 frequencies in the frequency range of $5 \mathrm{kHz}$ to $1 \mathrm{MHz}$ under incremental compression levels. A one-finger wearable force sensor FingerTPS by Pressure Profile Systems (Pressure Profile Systems Inc., LA, USA) was worn over the examiner's index finger. Before performing the test, the force sensor was calibrated using a load sensor and the customized software "Chameleon TVR", available with the PPS sensors. The constant pressure was applied to the electrical electrodes located over the tissue by the examiner placing her index finger over the electrodes and applying constant pressure. The applied pressure was approximately held constant within $5 \%$ bound by the examiner monitoring the pressure value from the Chameleon TVR software. This procedure was repeated at six force levels $(2.2-13.3 \mathrm{~N})$ for chicken and rat samples and at 3 force levels $(2.5-7.5 \mathrm{~N})$ for human subjects.

In the in-vitro experiment, two samples were excised from each chicken breast and one sample was excised from each rat breast. Therefore, twenty chicken samples excised from ten chicken breasts and two rat samples excised from two rat breasts were chosen for the experiments. Samples were cut in a cuboid form using a sharp cutter from the same parts of the animals' breast to assure consistency in results. The samples were $4 \mathrm{~cm}, 3.5 \mathrm{~cm}$ and $2 \mathrm{~cm}$ in length, width and thickness respectively. All the in-vitro measurements were performed within 1-2 hours after excitation at ambient temperature consistent with other studies [35]. For in-vitro bioimpedance measurements, two $\mathrm{Ag} / \mathrm{AgCl}$ pre-gelled disposable electrodes from Vermed (Vermed Inc., USA) were placed at two opposite sides of the cuboid samples (one electrode at the bottom and one electrode at the top sides of the samples). By means of a finger wearable capacitive pressure sensor on the examiner's finger, six constant force levels (2.2, 4.4, 6.7, 8.9, 11.1 and $13.3 \mathrm{~N}$ ) were applied to the electrodes. While keeping the pressure approximately constant at each level, the multi-frequency bioimpedance data was collected in three trials.

The in-vivo experiment was performed on left and right forearm and bicep of 11 healthy human subjects. The subjects were three females and eight males aged 24 to 45 . For the in-vivo experiment, two $\mathrm{Ag} / \mathrm{AgCl}$ electrodes were integrated into a probe shown in figure 1. This probe was designed in SolidWorks and prototyped by a 3D printer. Two $\mathrm{Ag} / \mathrm{AgCl}$ electrodes as well as six temperature sensors were integrated into this probe. However, the temperature sensors were not utilized in this study. The $\mathrm{Ag} / \mathrm{AgCl}$ electrodes were at $2 \mathrm{~cm}$ constant distance. For the sake of consistency in the results, the tests were conducted on the same places of both hands. For bicep and forearm tests, the probe was placed at a distance $8 \mathrm{~cm}$ from elbow and wrist, respectively. The in-vivo data collection procedure was the same as the in-vitro testing. The only difference was that in the in-vivo experiments, the force levels were chosen so that the pressure values were tolerable by human subjects. Thus, just three force levels $(2.5,5$ and $7.5 \mathrm{~N})$ were applied to the probe placed over the subjects' arms and biceps. The in-vitro and in-vivo admittances were measured at various force levels in three trials and recorded for further analysis.

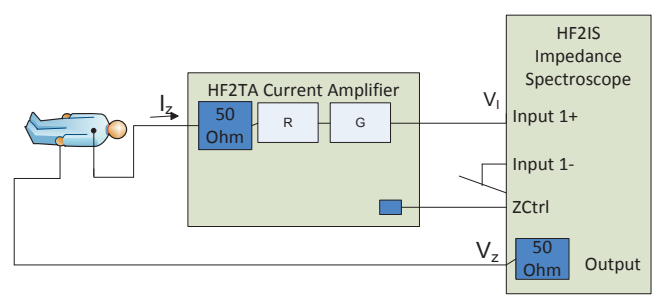

(a)

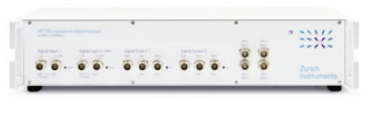

(b)

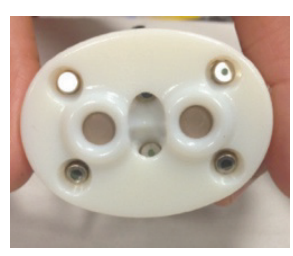

(c)

Fig.1: a) The electrical impedance setup b) The electrical impedance spectroscope (HF2IS) c) The designed and prototyped probe consisting of $2 \mathrm{Ag} / \mathrm{AgCl}$ electrodes and 6 temperature sensors (temperature electrodes were not used in this study)

Mechanical setup and procedure. Ten chicken breasts and two rat breasts (the same in-vitro breast samples used in the EIS experiment) were tested within five hours post-mortem interval, consistence with other studies [36-38]. To exclude the effect of fibers and, hence, anisotropic character of soft tissues in the study, samples were cut from transverse slices of the tissues in rectangular shape samples and compression testing was performed transversely perpendicular to the matrix of the fibers [2, 39-41]. Five samples from each chicken breast and one sample from each rat breast were prepared and compressed. The dimensions of the specimens were $15 \mathrm{~mm}, 8 \mathrm{~mm}$ and $6 \mathrm{~mm}$ in length, width and thickness respectively.

The mechanical characteristics of the chicken and rat samples were identified using an Electroforce 3200 (Bose Corp, Grand Prairie, MN) equipped with a $50 \mathrm{lbf}$ load cell (Sensotec 31E, Honeywell Corp, Columbus, OH). The samples were compressed with polished aluminum platens at $0.1 \mathrm{~N}$ preload. Before applying the preload, drops of water were added to keep the samples hydrated and reduce friction between samples and platens. While preloading the samples, the surface contacts between the samples and platens were also checked visually. When the specimens were contacting well with the platens, the loading and unloading cycle was started. Chen et al found linear stressstrain curves up to $5 \%$ strain for cylindrical diverse tissue species and nonlinear behavior - once the strain exceeded $10 \%$ [42]. Thus, all the samples were compressed to $10 \%$ strain at a strain rate of $0.002 \mathrm{~s}^{-1}$. The strain rate was kept constant when compressing the samples in order to exclude the effect of strain rate that would bring out the viscoelastic behavior of soft tissues [43]. The radial expansion of each specimen was visually monitored during the loading stage 
in order to detect the occurrence of elastic-bucking or other irregular deformation. The samples that had undergone irregular deformation or bucking were removed from the study.

The mechanical characterization of tissue constituents was evaluated by capturing responses of forearm and biceps of healthy subjects to a compressive loading. The Pressure Profile System Fingertip Sensor was used to obtain force values through indentation. The displacement data was acquired via Celesco Cable Extension Transducers (SP2 Series, Celesco Transducer Products Inc., Chatsworth, CA).

Forearm and bicep of eleven healthy human subjects (the same in-vivo subjects used in the EIS measurements) were mechanically characterized via fingertip force sensor and Celesco spring pot. The spring pot was fixed over a piece of wood, and the rob end was fixed over the fingertip force sensor worn by the operator. As the index finger was moving towards the phantom, force and displacement data were measured simultaneously in real time.

\section{Parameter fitting of Cole model in the form of admittance}

The admittance of human tissue can be expressed by the Cole equation in the form of admittance proposed by [29]:

$$
Y=G+j B=G_{\infty}+\frac{G_{0}-G_{\infty}}{1+\left(\frac{j f}{f_{y c}}\right)^{\alpha}}=\frac{1}{Z}
$$

where $\mathrm{Y}$ is the whole admittance, $\mathrm{G}$ is the conductance and B is the susceptance. $G_{0}$ is the admittance at zero driving frequency, $G_{\infty}$ is the admittance when the driving frequency is infinity, $f$ is the driving frequency, $f_{y c}$ is the frequency at which the imaginary part of the admittance reaches its maximum and $\alpha$ is the dispersion parameter.

As stated by Liu et al [29], the equation of the admittance forms the equation of a circle and thus plotting the imaginary part of admittance (susceptance) versus the real part of admittance (conductance) in a complex plane results in a circular arc as shown in figure $2 \mathrm{a}$.

Yang et al proposed an improved Least Absolute Deviation (LAD) method for data fitting and Cole feature extraction which is an alternative to the Least Square method [44]. The LAD method is an optimization method which minimizes the summation of the absolute errors which can be found by subtracting each data point from the fitted data [45].

$$
\text { Fitness Func. }=\min \sum_{i=1}^{n}\left|e_{i}\right|=\sum_{i=1}^{n}\left|Y_{\text {raw }}-Y_{\text {fitted }}\right|
$$

The BFGS quasi-newton method has been used in order to fit the data to the Cole model in the form of admittance [44]. This method aims to find the minimum of the fitness function shown in equation 2 considering the starting points as per Yang et al.

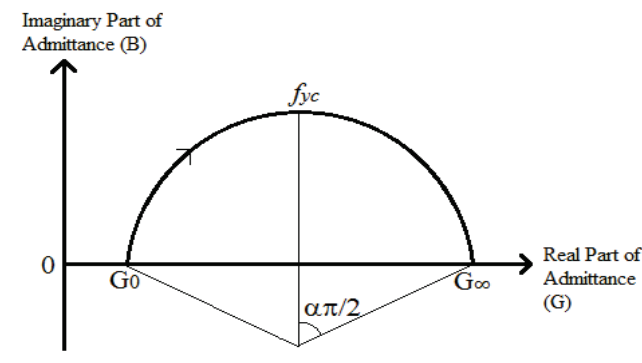

(a)

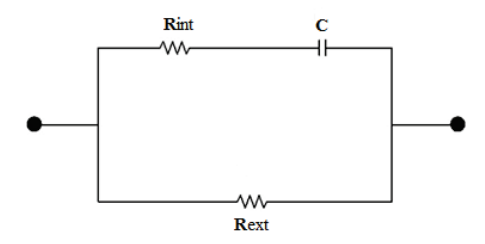

(b)

Fig.2: a) Imaginary part of admittance versus its real part (Cole arc). b) Cole model circuit.

Once the minimum of the equation 2 is found, the model is fitted to the Cole model and the four Cole features $\left(G_{0}, G_{\infty}\right.$, $\alpha$ and $f_{y c}$ ) are extracted by this method.

The Cole model, figure $2 \mathrm{~b}$, models the tissue as a circuit including one resistor in series with one capacitor, and both in parallel with a resistor. If the capacitor is ideal, it will correspond to the Debye case with $\alpha=1$, resulting in a full semicircle in the complex plane. Using a constant phase element (CPE) instead will result in a depressed circular arc with $\alpha \neq 1$. The resistor and capacitor which are in series mimic the intracellular resistance $\left(\mathrm{R}_{\text {int }}\right)$ and bulk membrane capacitance $\left(\mathrm{C}_{\mathrm{m}}\right)$ and the resistor in parallel with these two components mimics the extracellular resistance of tissue $\left(\mathrm{R}_{\text {ext }}\right)$. All these three Cole circuit parameters can be calculated by the following equations:

$$
\begin{gathered}
R_{\text {ext }}=R_{0} \\
R_{\text {int }}=\frac{R_{0} R_{\infty}}{R_{0}-R_{\infty}} \\
C_{m}=\frac{1}{2 \pi f_{c}\left(R_{\text {int }}+R_{\text {ext }}\right)}
\end{gathered}
$$

where $R_{0}=\frac{1}{G_{0}}, R_{\infty}=\frac{1}{G_{\infty}}$ and $f_{c}=f_{y c} \sqrt[\alpha]{\frac{G_{0}}{G_{\infty}}}$.

\section{Modulus of elasticity measurements and indentation theory}

In the present work, the indentation theories were applied for the in-vivo experiments considering soft tissue as an elastic layer bounded to a rigid body, which is deformed by a flat-ended cylindrical punch in the normal direction. The elastic modulus at each point of the indentation test was calculated using Hayes's theoretical solution [46]:

$$
E=\frac{P\left(1-v^{2}\right)}{2 \operatorname{Da\kappa }\left(v, \frac{a}{h}\right)}
$$


where $P$ is the indentation load, $D$ is the indentation displacement, $v$ is Poisson's ratio, $a$ is the radius of the indenter, $h$ is the thickness of the tissue and $\kappa$ is a factor which is a function of material properties and geometry of the tissue.

The average of stress distribution beneath the indenter was governed from the following equation $[46,47]$ :

$$
\bar{\sigma}=\frac{1}{\pi a^{2}} \int_{0}^{a} \frac{P}{2 \pi A \kappa}\left(a^{2}-\rho^{2}\right)^{-\frac{1}{2}} 2 \pi \rho d \rho
$$

where $\rho$ is the loaded location under the punch and the above equation is for $0 \leq \rho \leq a$. For $\rho>a, \sigma=0$. $\kappa$ is a factor which is a function of material properties and geometry of the tissue. The $\kappa$ values corresponding to relative indentation and aspect ratios of the current study were calculated by doing some interpolations on the provided tables for different indentation levels [48]. The elastic modulus of the tissue within $10 \%$ relative indentation was obtained from the slope of stress distribution versus relative indentation curve.

Regarding the unconfined compression testing of chicken and rat breast, elastic moduli of the samples were acquired from the slope of the engineering stress versus strain and Hooke's law.

$$
E=\frac{\sigma}{\varepsilon}
$$

\section{Results}

Findings for electrical properties of tissue, feature extraction of Cole model by LAD, mechanical properties of tissue and the correlation of the electrical features and the displacement of tissue are given below.

\section{Electrical properties of tissue extracted by LAD}

For each sample/subject, the measurements were performed at each pressure level at three trials. If the acquired data at each of these three trials were not similar to the the data of the two other trails due to any data acquisition error, that trial was excluded and the measured data of the two other trails were averaged. Knowing the applied current, the measured voltage and the sweeping frequency at each compression level and each trial, the admittance of the tested samples and subjects were calculated. For each sample/subject, the admittances of the three trials were then averaged within each compression level. Therefore, the average of raw admittance data of each sample/subject at each compression level were obtained. Fitting equation 1 to the averaged admittance Nyquist plots, resulted in the extracted Cole features $\left(G_{0}, G_{\infty}, a\right.$ and $\left.f_{y c}\right)$ within each pressure level. Cole circuit equivalent parameters $\left(R_{\text {ext }}, R_{\text {int }}\right.$ and $C_{m}$ ) were then calculated from equations 3 to 5 . Thus for all twenty chicken samples, two rat samples and eleven human subjects, $R_{\text {ext }}, R_{\text {int }}$ and $C_{m}$ were acquired at various pressure levels.
Table 1 represents the average of the Cole parameters of all subjects and samples at the first pressure levels $(2.5 \mathrm{~N}$ in human subjects and 2.2 $\mathrm{N}$ in chicken and rat samples) and their standard deviation.

\section{Mechanical properties of tissue}

The mechanical experimental results demonstrated an approximate linear correlation between stress and strain in the unconfined chicken and rat tissue samples and an approximate linear correlation between stress and indentation in the confined human subjects' tissues. Figure 3 illustrates the stress versus indentation of bicep of one of the human subjects. This figure shows that by increasing the magnitude of the applied stress to the tissue, the magnitude of the resultant strain increases and there is an approximately linear relationship between the applied stress and the strain in soft tissue. Similar behavior was observed in all samples and subjects. The elastic moduli of human subjects were acquired from the correlation of stress versus indentation and by incorporating equation 6 , and the elastic modulus of chicken and rat tissues were acquired from the correlation of stress versus strain and by incorporating equations 8 . The mean modulus of elasticity of ten chicken breasts and two rat tissues are $36902.79\left(\mathrm{~N} / \mathrm{m}^{2}\right)$ and 34810 $\left(\mathrm{N} / \mathrm{m}^{2}\right)$, respectively. The averaged elastic modulus of left and right forearm, left and right bicep of eleven human subjects are, $197641.6\left(\mathrm{~N} / \mathrm{m}^{2}\right)$ and $193221.9\left(\mathrm{~N} / \mathrm{m}^{2}\right)$, $165765.5\left(\mathrm{~N} / \mathrm{m}^{2}\right)$ and $162921.7\left(\mathrm{~N} / \mathrm{m}^{2}\right)$, respectively. These acquired elastic moduli will be used in the following section for correlating the changes of electrical properties and tissue displacements.

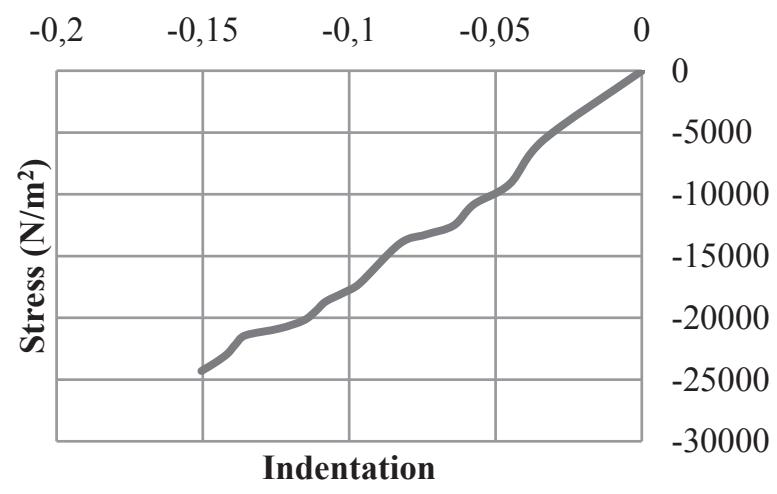

Fig.3: Stress versus indentation of in-vivo bicep of one subject.

Tissue displacement-dependent features analysis

Based on the modulus of elasticity of each type of tissue calculated in the previous section, the amount of displacement at each pressure level was calculated. The displacement of the unconfined tissues, chicken and rat, were measured by the Hooke's law and the displacement of the confined human subjects were calculated by the indentation theory. The extracted Cole features and subsequently the Cole circuit equivalent parameters at each pressure level were obtained in previous sections. The 
Table 1. Averaged Cole parameters by LAD method at the first pressure level and the standard deviations

\begin{tabular}{cccc}
\hline \multirow{2}{*}{ Samples or Subjects } & \multicolumn{3}{c}{ Fitting Results } \\
\cline { 2 - 4 } & $\mathrm{R}_{\text {ext }}(\Omega)$ & $\mathrm{R}_{\text {int }}(\Omega)$ & $\mathrm{C}_{\mathrm{m}}(\mathrm{nF})$ \\
\hline Chicken Breasts & $537.85 \pm 23.19$ & $512.34 \pm 24.32$ & $7.91 \pm 0.50$ \\
Rat Breasts & $565.54 \pm 18.19$ & $675.83 \pm 98.93$ & $30.7 \pm 14.9$ \\
Left Forearms & $2612508.42 \pm 359731.87$ & $986.46 \pm 189.34$ & $16.46 \pm 6.19$ \\
Right Forearms & $2125924.31 \pm 189807.62$ & $970.02 \pm 183.89$ & $18.45 \pm 8.99$ \\
Left Biceps & $7577699.16 \pm 180562.62$ & $1386.3 \pm 299.1259$ & $14.83 \pm 7.65$ \\
Right Biceps & $7563298.20 \pm 121059.83$ & $1252.9 \pm 437.72$ & $15.26 \pm 8.53$ \\
\hline
\end{tabular}

extracellular resistance, intracellular resistance and membrane capacitance of each subject and sample were normalized to their corresponding values at the first compression level. These normalized parameters measured at each pressure level were then averaged and plotted against that pressure corresponding displacement in figures 4-9. These figures illustrate a linear relationship between the normalized Cole parameters and the displacement of tissue.

As figure 4 illustrates, at the maximum displacement of chicken tissue when the maximum force, $3 \mathrm{lb}$, is applied to the tissue samples, the normalized extracellular resistance increases $45 \%$ relative to its first value. Normalized intracellular resistance has a decrease of $20 \%$ and normalized membrane capacitance has an increase of $48 \%$ in chicken tissue. The changes in rat tissue includes an increase of $6 \%$ in normalized extracellular resistance, $7 \%$ decrease of normalized intracellular resistance and $17 \%$ increase of normalized membrane capacitance as observed in figure 5. Figures 6-9 indicate that at the maximum force applied to the human subjects, $7.5 \mathrm{~N}$, the normalized extracellular resistance has an increase of $39.7 \%$ and $38.5 \%$ in left and right forearm and an increase of 294\% and 297\% in left and right bicep. At this point, the normalized intracellular resistance increases $8.8 \%$ and $8.9 \%$ in left and right forearm and $12.4 \%$ and $12.5 \%$ in left and right bicep. The decrease of normalized membrane capacitance in left and right forearm is $11.8 \%$ and $12.3 \%$ and in left and right bicep are $26.9 \%$ and $24.0 \%$ respectively.

\section{Discussion and Conclusion}

This study quantified the effect that compression has on the bioimpedance of soft tissue. Two types of experiments were conducted in this study, in-vitro and in-vivo. The results of this study show a linear correlation between the changes of electrical properties of tissue and its displacement in both in-vivo and in-vitro results. The Pearson products of the linear relationships $(0.918<p<0.999)$ indicate that the electrical properties of soft tissue correlate well to the mechanical changes as samples and subjects are compressed. The difference in slopes and intercepts of the fitted models to the chicken, rat and human tissues data (figures 4 to 9 ) indicates that each type of tissue responds differently to compression, suggesting that electromechanical relation of tissue may be affected by its type and structure.

Because of the high impedance of cell's membrane in lower frequencies, current flows between the cells and through the extracellular space, therefore the resistance measured at lower frequencies is mostly an indicator of the extracellular resistance. While at higher frequencies permeability of the cells' membrane increases, which allows current to cross the cell membrane, and therefore both intracellular and extracellular resistances of tissue influence the measured resistance. The Cole model of biological tissue also supports this idea by the fact that open circuit occurs at lower frequencies at branches containing capacitance and current flows only through the resistor called extracellular resistance and at higher frequencies, the capacitor short circuit occurs and current flows through both resistances in parallel. Accordingly, at higher frequencies, the equivalent resistance is comprised of two parallel resistors, which have a lower value compared to each resistor's value. Therefore, in general, the value of resistance at higher frequencies is less than lower frequencies regardless of type of tissue. This has also been observed in previous experiments by Boris-Reymond (1848-1860) [49] and in the current study, by the fact that the equivalent resistances at lower frequencies in chicken and rat breast are $537.85 \Omega$ and $565.54 \Omega$ and at higher frequencies are $262.39 \Omega$ and $307.89 \Omega$ respectively.

Based on the values of extracellular resistances in this study, it is postulated that the lower extracellular resistance reflects a narrower extracellular space in any type of tissue. Koshini et al also explained the increase in $R_{\text {ext }}$ as a result of reduction in the extracellular volume [50]. The values at the first pressure level listed in table 1 indicate that the extracellular resistance of rat is higher compared to chicken, which according to this postulation it may be caused by the lower extracellular area between rat cells where current faces less space to travel through. This idea is supported by another experimental observation in which squamous epithelium tissue with more tightly packed cells shows higher resistance in lower frequencies compared to columnar and transitional epithelia, which have wider extracellular space [26]. The idea that the value of extracellular resistance is influenced by the space between 
cells, may infer that applying pressure reduces the extracellular space and subsequently the extracellular resistance of tissue increases. This hypothesis is reflected in the extracellular behavior under compression in both in-vivo and in-vitro results (part a's of figures 4 to 9).

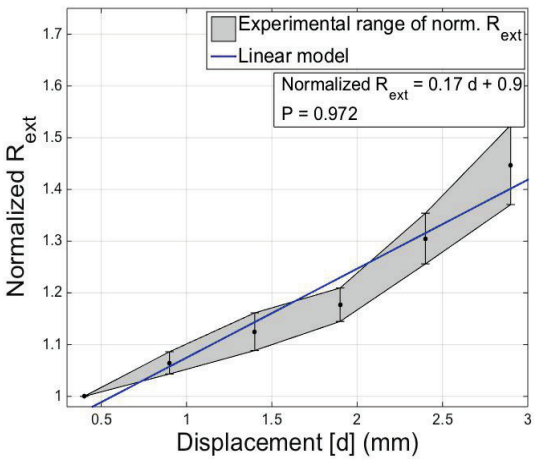

(a)

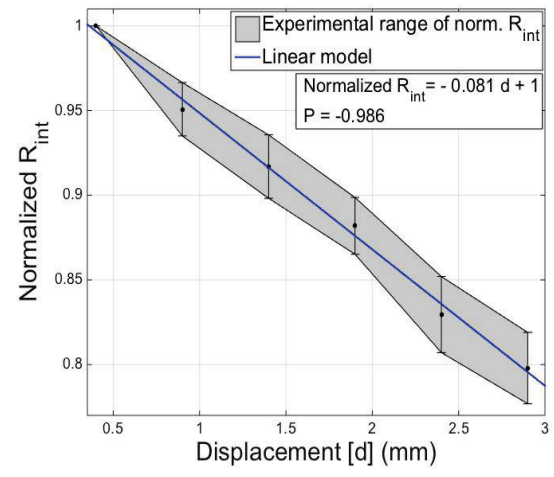

(b)

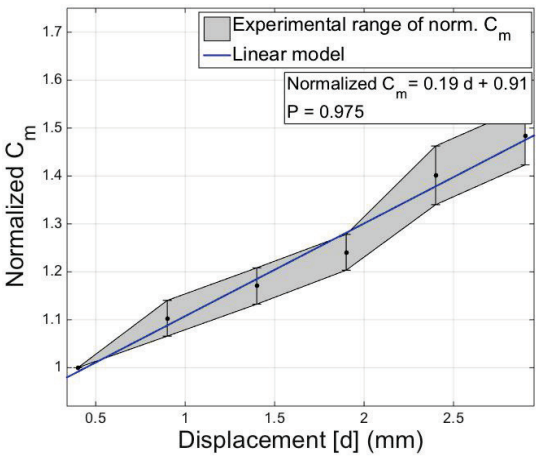

(c)

Fig.4: Chicken breast tissue, the correlation of compression-dependent Cole parameters normalized to their values at the first pressure level and tissue displacements. (a) compression-dependent extracellular resistance versus tissue displacement. The correlation is: Normalized $R_{\text {ext }}=0.17 w+0.9$ and the Pearson product of the linear relationship is 0.972 (b) compression-dependent intracellular resistance versus tissue displacement. The correlation is: Normalized $R_{\text {int }}=-0.081 w+1$ and the Pearson product of the linear relationship is -0.986 (c) compression-dependent membrane capacitance versus tissue displacement. The correlation is: Normalized $C_{m}=0.19 w+0.91$ and the Pearson product of the linear relationship is 0.975 .

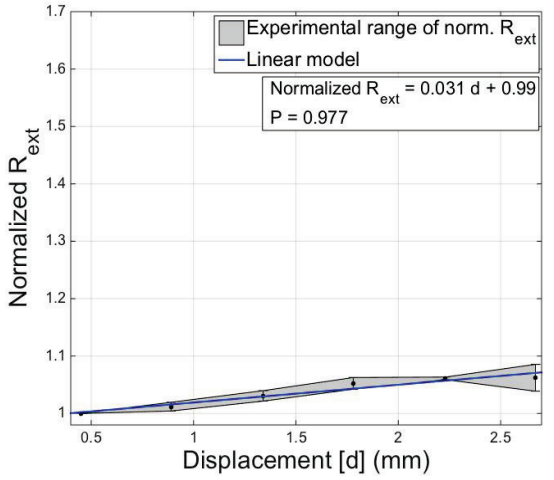

(a)

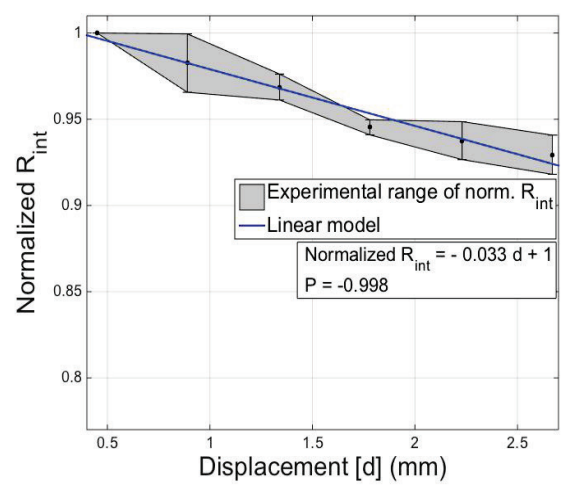

(b)

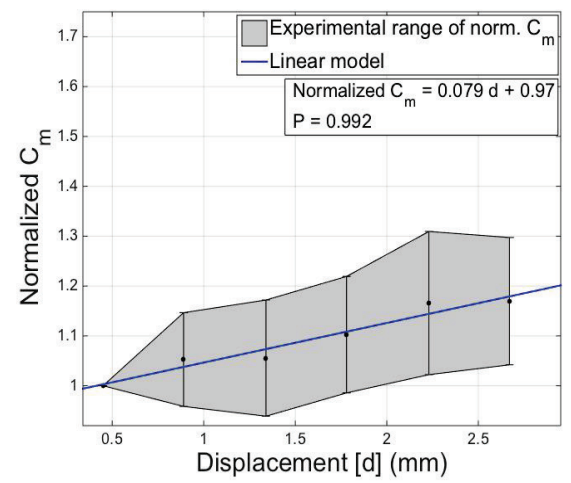

(c)

Fig.5: Rat breast tissue, the correlation of compression-dependent Cole parameters normalized to their values at the first pressure level and tissue displacements. (a) compression-dependent extracellular resistance versus tissue displacement. The correlation is: Normalized $R_{\text {ext }}=0.031 w+0.99$ and the Pearson product of the linear relationship is 0.977 (b) compression-dependent intracellular resistance versus tissue displacement. The correlation is: Normalized $R_{\text {int }}=-0.033 w+1$ and the Pearson product of the linear relationship is -0.998 (c) compression-dependent membrane capacitance versus tissue displacement. The correlation is: Normalized $C_{m}=0.079 w+0.97$ and the Pearson product of the linear relationship is 0.992 .

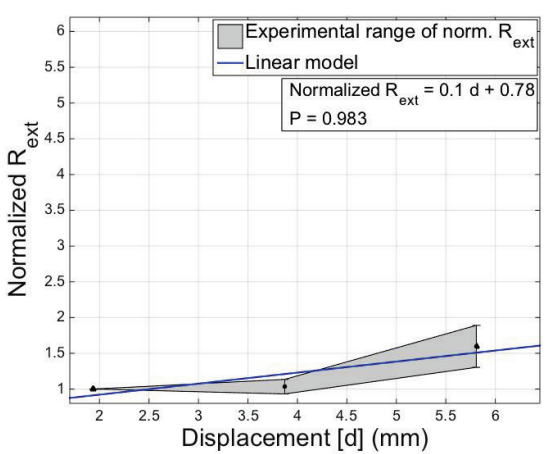

(a)

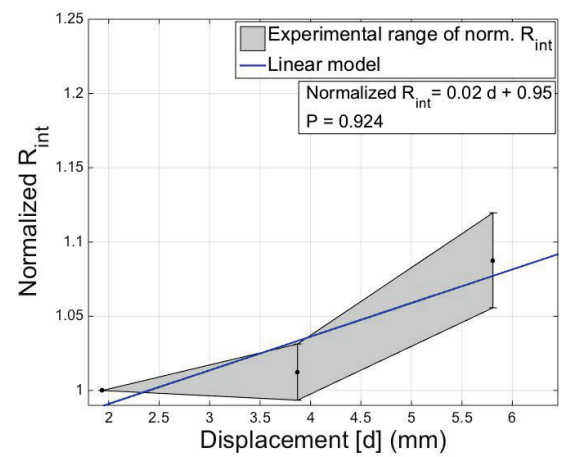

(b)

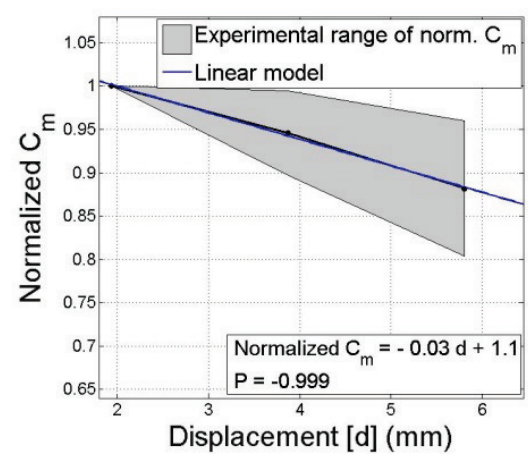

(c)

Fig.6: Left Forearm Tissue, the correlation of compression-dependent Cole parameters normalized to their values at the first pressure level and tissue displacements. (a) compression-dependent extracellular resistance versus tissue displacement. The correlation is: Normalized $R_{\text {ext }}=0.1 \quad w+0.78$ and the Pearson product of the linear relationship is 0.983 (b) compression-dependent intracellular resistance versus tissue displacement. The correlation is: Normalized $R_{\text {int }}=0.023 w+0.95$ and the Pearson product of the linear relationship is 0.924 (c) compression-dependent membrane capacitance versus tissue displacement. The correlation is: Normalized $C_{m}=-0.031 w+1.1$ and the Pearson product of the linear relationship is -0.999 . 


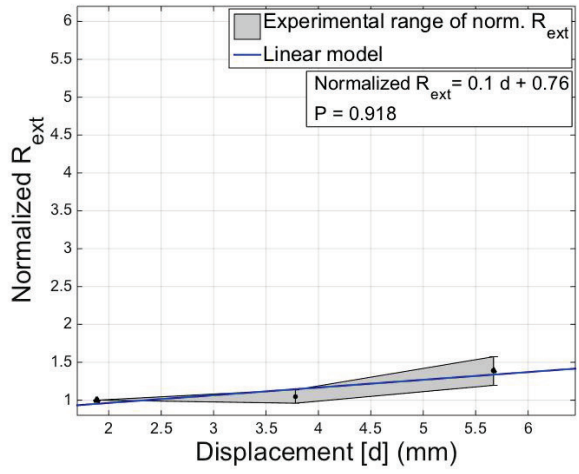

(a)

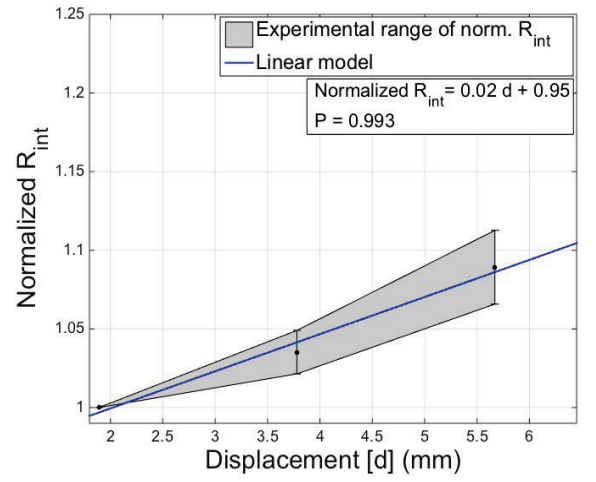

(b)

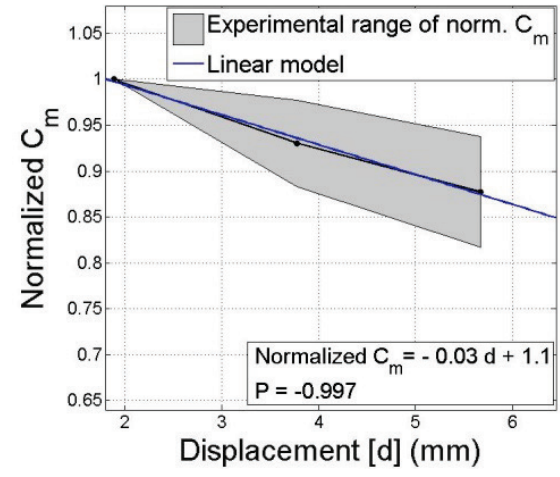

(c)

Fig.7: Right Forearm Tissue, the correlation of compression-dependent Cole parameters normalized to their values at the first pressure level and tissue displacements. (a) compression-dependent extracellular resistance versus tissue displacement. The correlation is: Normalized $R_{\text {ext }}=0.1 w+0.76$ and the Pearson product of the linear relationship is 0.918 (b) compression-dependent intracellular resistance versus tissue displacement. The correlation is: Normalized $R_{\text {int }}=0.024 w+0.95$ and the Pearson product of the linear relationship is 0.993 (c) compression-dependent membrane capacitance versus tissue displacement. The correlation is: Normalized $C_{m}=-0.032 w+1.1$ and the Pearson product of the linear relationship is -0.997 .

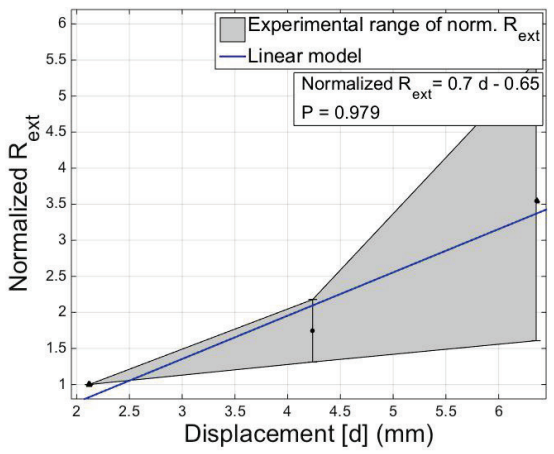

(a)

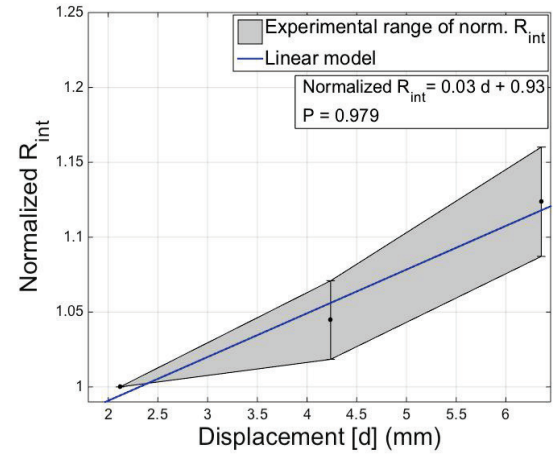

(b)

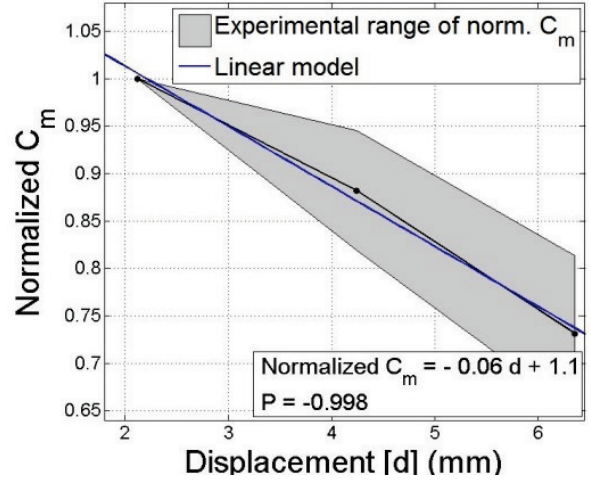

(c)

Fig.8: Left Bicep Tissue, the correlation of compression-dependent Cole parameters normalized to their values at the first pressure level and tissue displacements. (a) compression-dependent extracellular resistance versus tissue displacement. The correlation is: Normalized $R_{\text {ext }}=0.69$ w-0.65 and the Pearson product of the linear relationship is 0.979 (b) compression-dependent intracellular resistance versus tissue displacement. The correlation is: Normalized $R_{\text {int }}=0.029 w+0.93$ and the Pearson product of the linear relationship is 0.969 (c) compression-dependent membrane capacitance versus tissue displacement. The correlation is: Normalized $C_{m}=-0.063 w+1.1$ and the Pearson product of the linear relationship is -0.998 .

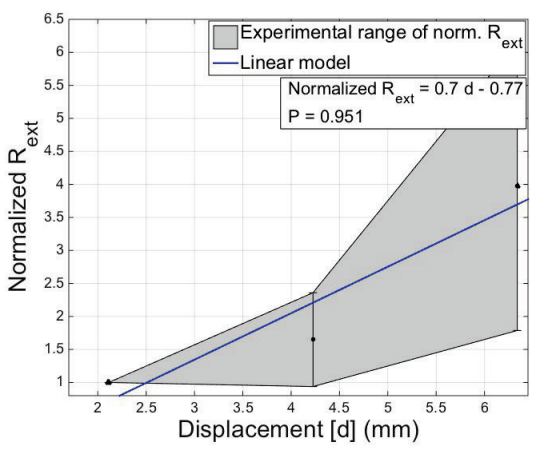

(a)

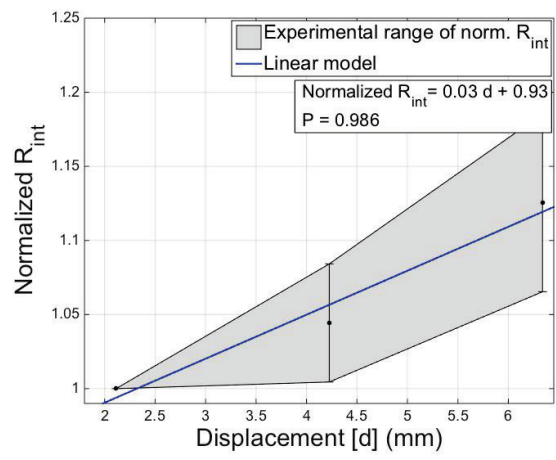

(b)

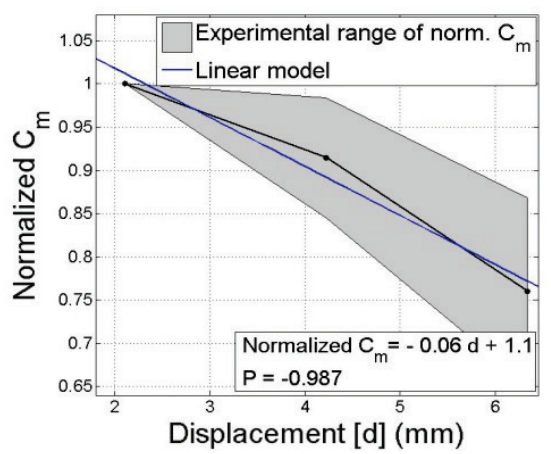

(c)

Fig.9: Right Bicep Tissue, the correlation of compression-dependent Cole parameters normalized to their values at the first pressure level and tissue displacements. (a) compression-dependent extracellular resistance versus tissue displacement. The correlation is: Normalized $R_{\text {ext }}=0.7$ w- 0.77 and the Pearson product of the linear relationship is 0.951 (b) compression-dependent intracellular resistance versus tissue displacement. The correlation is: Normalized $R_{\text {int }}=0.03 w+0.93$ and the Pearson product of the linear relationship is 0.986 (c) compression-dependent membrane capacitance versus tissue displacement. The correlation is: Normalized $C_{m}=-0.057 w+1.1$ and the Pearson product of the linear relationship is -0.987. 
Another finding of this study was that while the changes in extracellular resistance have the same pattern in both invivo and in-vitro tests, the intracellular resistance and membrane capacitance show different behavior in in-vivo and in-vitro experiments. The in-vivo intracellular resistance increases as pressure is increased. The reason of increase in $R_{\text {int }}$ could be the reduced intracellular space. Because the reduced intracellular space resists the current flowing cross the cells. This difference between the in-vivo and the invitro results may be because in the in-vitro tests, the samples are collected after the animals' death while in the in-vivo study, the subjects are alive and blood flow exists. When the blood supply is cut off in a tissue, the activity of ion pumps reduces and thus the distribution of ions and fluids between intracellular and extracellular spaces changes, which leads to cell swelling [51]. Cell swelling increases the intracellular volume and this could be a reason of reduction in the intracellular resistance in the in-vitro study. Cell swelling also reduces the extracellular pathways and makes them narrower, which is another evidence for the increase in the extracellular resistance [52]. Cell swelling and also release of Lysosomal enzymes in the cell, causes rupture in cell membrane [53]. In the post-mortem specimens, the cell membrane starts to deteriorate. Thus the cytosol leaves the cell and is mixed with the extracellular fluid [52]. This procedure is accelerated by applying pressure. The reduction in the intracellular fluid and the increase of the extracellular fluid (mixing the intracellular and extracellular fluid at the extracellular space) caused by deterioration of cell membrane and applying pressure might be another explanation of reduction in $R_{\text {int }}$ and increase in $R_{\text {ext }}$. Demou also has mentioned the possibility of reshaping of cells from spherical to oblong orientation which may result in an increase or decrease in electrical properties of tissue [54].

Membrane capacitance in in-vitro experiments increases. The value of capacitance is proportional to the area of cell membranes and inversely proportional to the separation between sheets. Cell swelling and applying pressure increases the surface [51] and the cell membrane becomes thinner as a result of inflation [50]. Thus, a possible explanation for the reduction in membrane capacitance might be the decreased distance between two sheets of cell membrane and increased surface of these sheets. However, membrane capacitance decreases as the applied pressure to the in-vivo subjects is increased, which according to Dodde et al might be the result of contacting the cell membranes [25].

In this study, the changes in electrical properties of living tissue were also compared contra-laterally. The results illustrate the same behavior and the same linear correlation in contra-lateral parts of human subjects. This observation suggests the idea that the correlation of electrical-mechanical properties of one part of body is the same as its contra-lateral part unless there is an abnormality in one of the contra-lateral parts. This comparison of electrical-mechanical correlation of contra-lateral parts of the body may be considered as a method for diagnosis of tumor or any abnormalities within tissue.

The results of in-vivo and in-vitro strengthen the fact that EIS measurements of in-vitro experiments do not represent an exact in-vivo environment. This idea insists on performing more experiments in-vivo rather than in-vitro in order to get more reliable results in the field of electrical impedance spectroscopy. One possibility for different behavior of in-vivo and in-vitro results would be the blood flow in the in-vivo case. The flowing blood in the living tissue is the most important factor influencing the tissue impedance. Since there is no blood flowing in dead tissue, the changes of its impedance properties do not follow the same pattern as the living tissue.

However, the findings of the current study do not fully support the previous research by Dodde [25]. The reason for this inconsistency may arise from the difference in the experiment protocols of these two studies, thus this is an important issue for future research. Further research should be done to investigate the effect of pressure on electrical properties of tissue both in-vivo and in-vitro.

This study also suggests the idea of modifying the Cole model. In the Cole model, the tissue equivalent resistance and capacitance values are constant while according to this study a modified Cole model should be used in which the parameters are dependent on tissue displacement and elasticity.

There were also some limitations in this study, which should be eliminated during further experiments. In the invitro experiment, the tissues were tested on a firm foundation and samples were not supported by surrounding tissue and bone while there were not the same conditions in the in-vivo tests. Another limitation of this study is that all the experiments were performed in the room temperature and there is a possibility that the room temperature was not constant during the experiments. As shown by Schwan and Foster, the electrical conductivity of tissue is dependent on temperature [55].

\section{Acknowledgements}

Financial support of this project was provided by the Natural Sciences and Engineering Council of Canada (NSERC). The authors would like to thank Ehsan Daneshi Kohan and Shima Zaeimdar for their help in progress of this project.

\section{References}

1. T. Krouskop, T. Wheeler, and F. Kallel, "Elastic moduli of breast and prostate tissues under compression," Ultrason. Imaging, vol. 20, no. 4, pp. 260-274, 1998. http://dx.doi.org/10.1177/016173469802000403

2. J. J. O'Hagan and A. Samani, "Measurement of the hyperelastic properties of tissue slices with tumour inclusion," Phys. Med. Biol., vol. 53, no. 24, pp. 7087-106, 2008. http://dx.doi.org/10.1088/0031-9155/53/24/006 
3. P. Wellman and R. Howe, "Breast tissue stiffness in compression is correlated to histological diagnosis," Harvard BioRobotics, pp. 1-15, 1999.

http://www.biorobotics.harvard.edu/pubs/1999/mechprops.pdf

4. J. Jossinet, "The impedivity of freshly excised human breast tissue," Physiol. Meas., vol. 19, no. 1, pp. 61-75, 1998. http://dx.doi.org/10.1088/0967-3334/19/1/006

5. F. Kallel and J. Ophir, "Tissue mechanical attributes imaging: principles and methods," in Computer-Based Medical Systems. CBMS 2000, pp. 147-159, 2000.

6. J. Bercoff, S. Chaffai, and M. Tanter, "In vivo breast tumor detection using transient elastography," Ultrasound Med. Biol., vol. 29 , no. 10, pp. 1387-1396, 2003. http://dx.doi.org/10.1016/S0301-5629(03)00978-5

7. A. B. Nover, S. Jagtap, W. Anjum, H. Yegingil, W. Y. Shih, W.-H. Shih, and A. D. Brooks, "Modern breast cancer detection: a technological review," Int. J. Biomed. Imaging, vol. 2009, p. 902326, Jan. 2009. http://dx.doi.org/10.1155/2009/902326

8. Y. C. Fung, Biomechanics: Mechanical Properties of living tissues. New York: New York: Springer-Verlag, 1993. http://dx.doi.org/10.1007/978-1-4757-2257-4

9. [9] A. A. Fischer, "Pressure algometry over normal muscles. Standard values, validity and reproducibility of pressure threshold," Pain, vol. 30, pp. 115-126, 1987. http://dx.doi.org/10.1016/0304-3959(87)90089-3

10. B. S. Garra, E. I. Cespedes, J. Ophir, S. R. Spratt, R. A. Zuurbier, C. M. Magnant, and M. F. Pennanen, "Elastography of breast lesions: initial clinical results," Radiology, vol. 202, pp. 79-86, 1997.

http://dx.doi.org/10.1148/radiology.202.1.8988195

11. T. L. Chenevert, A. R. Skovoroda, M. O'Donnell, and S. Y. Emelianov, "Elasticity reconstructive imaging by means of stimulated echo MRI," Magn. Reson. Med., vol. 39, pp. 482490, 1998. http://dx.doi.org/10.1002/mrm.1910390319

12. W. C. Hayes, L. M. Keer, G. Herrmann, and L. F. Mockros, "A mathematical analysis for indentation tests of articular cartilage," J. Biomech., vol. 5, pp. 541-551, 1972. http://dx.doi.org/10.1016/0021-9290(72)90010-3

13. T. A. Krouskop, T. M. Wheeler, and F. Kallel, "Elastic moduli of breast and prostate tissues under compression," Ultrason. Imaging, vol. 20, no. 4, pp. 260-274, 1998. http://dx.doi.org/10.1177/016173469802000403

14. A. P. Sarvazyan, A. R. Skovoroda, and Y. P. Pyt'ev, "Mechanical introscopy-a new modality of medical imaging for detection of breast and prostate cancer," Proc. Eighth IEEE Symp. Comput. Med. Syst., pp. 4-5, 1995. http://dx.doi.org/10.1109/cbms.1995.465455

15. M. Zhang, Y. P. Zheng, and A. F. T. Mak, "Estimating the effective Young's modulus of soft tissues from indentation tests-nonlinear finite element analysis of effects of friction and large deformation," Med. Eng. Phys., vol. 19, no. 6, pp. 512-517, 1997. http://dx.doi.org/10.1016/S1350-4533(97)00017-9

16. Y. C. Fung, Biomechanics: Mechanical Properties of living tissues. New York: Springer-Verlag, 1993. http://dx.doi.org/10.1007/978-1-4757-2257-4

17. S. Laufer, A. Ivorra, V. E. Reuter, B. Rubinsky, and S. B. Solomon, "Electrical impedance characterization of normal and cancerous human hepatic tissue," Physiol. Meas., vol. 31, pp. 995-1009, 2010. http://dx.doi.org/10.1088/0967-3334/31/7/009

18. T. Morimoto, S. Kimura, Y. Konishi, K. Komaki, T. Uyama, Y. Monden, D. Y. Kinouchi, and D. T. Iritani, "A Study of the Electrical Bio-impedance of Tumors," J. Invest. Surg., vol. 6, pp. 25-32, 1993.

http://dx.doi.org/10.3109/08941939309141189

19. J. Jossinet, "Variability of impedivity in normal and pathological breast tissue," Med. Biol. Eng. Comput., vol. 34, pp. 346-350, 1996. http://dx.doi.org/10.1007/BF02520002

20. A. Keshtkar, A. Keshtkar, and R. H. Smallwood, "Electrical impedance spectroscopy and the diagnosis of bladder pathology," Physiol. Meas., vol. 27, pp. 585-596, 2006. http://dx.doi.org/10.1088/0967-3334/27/7/003

21. B. H. Brown, J. Tidy, K. Boston, A. D. Blackett, and F. Sharp, "Tetrapolar measurement of cervical tissue structure using impedance spectroscopy," Proc. 20th Annu. Int. Conf. IEEE EMBC, vol. 6, pp. 2886-2889, 1998. http://dx.doi.org/10.1109/iembs.1998.746089

22. C. A. Gonzalez-Correa, "Electrical bioimpedance readings increase with higher pressure applied to the measuring probe," Physiol. Meas., vol. 26, no. 2, pp. 39-47, 2005. http://dx.doi.org/10.1088/0967-3334/26/2/004

23. B. S. Kim, D. Isaacson, H. Xia, T.-J. Kao, J. C. Newell, and G. J. Saulnier, "A method for analyzing electrical impedance spectroscopy data from breast cancer patients," Physiol. Meas., vol. 28, pp. S237-S246, 2007. http://dx.doi.org/10.1088/0967-3334/28/7/S17

24. K. S. Cole, "Permeability and Impermeability of cell membranes for ions," Cold Spring Harb. Symp. Quant. Biol., vol. 8, pp. 110-122, 1940. http://dx.doi.org/10.1101/SQB.1940.008.01.013

25. R. Dodde, J. Bull, and A. Shih, "Bioimpedance of soft tissue under compression," Physiol. Meas., vol. 33, pp. 1095-1109, 2012. http://dx.doi.org/10.1088/0967-3334/33/6/1095

26. C. Gonzalez-Correa, "Electrical bioimpedance readings increase with higher pressure applied to the measuring probe," Physiol. Meas., vol. 26, no. 2, pp. 39-47, 2005. http://dx.doi.org/10.1088/0967-3334/26/2/004

27. B. Belmont, R. Dodde, and A. Shih, "Impedance of tissuemimicking phantom material under compression," J. Electr. Bioimpedance, vol. 4, pp. 2-12, 2013. http://dx.doi.org/10.5617/jeb.443

28. J. Jossinet, C. Trillaud, and S. Chesnais, "Impedance changes in liver tissue exposed in vitro to high-energy ultrasound," Physiol. Meas., vol. 26, pp. S49-S58, 2005. http://dx.doi.org/10.1088/0967-3334/26/2/005

29. R. Liu, X. Dong, F. Fu, F. You, and X. Shi, "Multi-frequency parameter mapping of electrical impedance scanning using two kinds of circuit model," Physiol. Meas., vol. 28, no. 7, pp. 85100, 2007. http://dx.doi.org/10.1088/0967-3334/28/7/S07

30. K. S. Cole, "Electrical impedance of suspensions of spheres," J. Gen. Physiol., vol. 12, no. 1, pp. 29-36, 1928. http://dx.doi.org/10.1085/jgp.12.1.29

31. K. S. Cole and R. H. Cole, "Electrical impedance of arbacia eggs," J. Gen. Physiol., vol. 19, no. 4, pp. 625-632, 1936. http://dx.doi.org/10.1085/jgp.19.4.625 
32. F. Golnaraghi and P. K. Grewal, "Pilot study: electrical impedance based tissue classification using support vector machine classifier," IET Sci. Meas. Technol., vol. 8, pp. 579587, 2014. http://dx.doi.org/10.1049/iet-smt.2013.0087

33. O. G. Martinsen and S. Grimnes, Bioimpedance and bioelectricity basics. London: Academic Press, 2011.

34. P. K. Grewal, M. Shokoufi, J. Liu, K. Kalpagam, and K. S. Kohli, "Electrical characterization of bolus material as phantom for use in electrical impedance and computed tomography fusion imaging," J. Electr. Bioimpedance, vol. 5, no. 1, pp. 34-39, 2014. http://dx.doi.org/10.5617/jeb.781

35. B. Rigaud, L. Hamzaoui, M. R. Frikha, N. Chauveau, and J.-P. Morucci, "In vitro tissue characterization and modelling using electrical impedance measurements in the $100 \mathrm{~Hz}-10 \mathrm{MHz}$ frequency range," Physiol. Meas., vol. 16, no. 3A, pp. A15A28, 1995. http://dx.doi.org/10.1088/0967-3334/16/3A/002

36. R. Sinkus, J. Bercoff, M. Tanter, J.-L. Gennisson, C. ElKhoury, V. Servois, A. Tardivon, and M. Fink, "Nonlinear viscoelastic properties of tissue assessed by ultrasound," IEEE Trans. Ultrason. Ferroelectr. Freq. Control, vol. 53, no. 11, pp. 2009-2018, 2006. http://dx.doi.org/10.1109/TUFFC.2006.141

37. S. Maclean, "Brain tissue: Analysis of mechanical properties," URL: http://hdl.handle.net/1811/44968. Ohio State University, 2010.

38. K. K. Darvish and J. R. Crandall, "Nonlinear viscoelastic effects in oscillatory shear deformation of brain tissue," Med. Eng. Phys., vol. 23, no. 9, pp. 633-45, 2001. http://dx.doi.org/10.1016/S1350-4533(01)00101-1

39. C. J. Sparrey, "The role of constituent materials in spinal cord biomechanics," PhD thesis, University of California, 2008.

40. T. Kaster, I. Sack, and a Samani, "Measurement of the hyperelastic properties of ex vivo brain tissue slices," J. Biomech., vol. 44, no. 6, pp. 1158-63, 2011. http://dx.doi.org/10.1016/j.jbiomech.2011.01.019

41. J. J. O'Hagan and A. Samani, "Measurement of the hyperelastic properties of 44 pathological ex vivo breast tissue samples.," Phys. Med. Biol., vol. 54, no. 8, pp. 2557-69, 2009. http://dx.doi.org/10.1088/0031-9155/54/8/020

42. E. Chen, J. Novakofski, K. Jenkins, and W. Brien, "Young's modulus measurements of soft tissues with application to elasticity imaging," IEEE Trans. Ultrasonics, Ferroelectrics, and Frequency Control, vol. 43, no. 1, pp. 191-194, 1996. http://dx.doi.org/10.1109/58.484478

43. C. U. Devi, R. S. Bharat Chandran, R. M. Vasu, and A. K. Sood, "Measurement of visco-elastic properties of breasttissue mimicking materials using diffusing wave spectroscopy," J. Biomed. Opt., vol. 12, no. 3, p. 034035 , 2007. http://dx.doi.org/10.1117/1.2743081
44. Y. Yang, W. Ni, and Q. Sun, "Improved Cole parameter extraction based on the least absolute deviation method," Physiol. Meas., vol. 34, no. 10, pp. 1239-1252, 2013. http://dx.doi.org/10.1088/0967-3334/34/10/1239

45. K. Chen, Z. Ying, H. Zhang, and L. Zhao, "Analysis of least absolute deviation," Biometrika, vol. 95, no. 1, pp. 107-122, 2008. http://dx.doi.org/10.1093/biomet/asm082

46. W. Hayes, L. Keer, G. Herrmann, and L. Mockros, "Mathematical analysis for indentation tests of articular cartilage," J. Biomech., vol. 5, no. 5, pp. 541-551, 1972. http://dx.doi.org/10.1016/0021-9290(72)90010-3

47. I. A. N. N. Sneddon, "The relation between load and penetration in the axysmmetric Boussinesq problem for a punch of arbitrary profile," Int. J. Eng. Sci., vol. 3, no. 638, pp. 47-57, 1965. http://dx.doi.org/10.1016/0020-7225(65)90019-4

48. M. Zhang, Y. P. Zheng, and A. F. T. Mak, "Estimating the effective Young's modulus of soft tissues from indentation tests - nonlinear finite element analysis of effects of friction and large deformation," Med. Eng. Phys., vol. 19, no. 6, pp. 512-517, 1997. http://dx.doi.org/10.1016/S1350-4533(97)00017-9

49. E. McAdams and J. Jossinet, "Tissue impedance: a historical overview," Physiol. Meas., vol. 16, pp. A1-A13, 1995. http://dx.doi.org/10.1088/0967-3334/16/3A/001

50. Y. Konishi, T. Morimoto, Y. Kinouchi, T. Iritani, and Y. Monden, "Electrical properties of extracted rat liver tissue," Res. Exp. Med., vol. 195, pp. 183-192, 1995. http://dx.doi.org/10.1007/BF02576787

51. D. Haemmerich, R. Ozkan, S. Tungjitkusolmun, J. Z. Tsai, D. M. Mahvi, S. T. Staelin, and J. G. Webster, "Changes in electrical resistivity of swine liver after occlusion and post mortem," Med. Biol. Eng. Comput., vol. 40, no. 1, pp. 29-33, 2002. http://dx.doi.org/10.1007/BF02347692

52. D. Miklavacic, N. Pavselj, and F. X. Hart, Wiley encyclopedia of biomedical engineering. 2006.

53. J. L. Farber, K. R. Chien, and S. J. Mittnacht, "Myocardial Ischemia: the Pathogenesis of irreversible cell Injury in Ischemia," Am. J. Pathol., vol. 102, pp. 271-281, 1981.

54. Z. Demou, "Gene expression profiles in 3D tumor analogs indicate compressive strain differentially enhances metastatic potential," Ann. Biomed. Eng., vol. 38, no. 11, pp. 3509-3520, 2010. http://dx.doi.org/10.1007/s10439-010-0097-0

55. H. Schwan and K. Foster, "RF-field interactions with biological systems: electrical properties and biophysical mechanisms," Proc. IEEE, vol. 68, no. 1, pp. 104-113, 1980. http://dx.doi.org/10.1109/PROC.1980.11589 